\title{
O processo Clinical Caritas de Jean Watson na assistência de enfermagem brasileira: uma revisão sistemática
}

\section{The process in Clinical Care Caritas' Jean Watson in brazilian of nursing: a systematic review}

\section{El proceso Clinical Caritas de Jean Watson en la enfermería brasileña : una revisión sistemática}

\author{
${ }^{1}$ Nirvania V. Carvalho; ${ }^{2}$ Dinah Sá Rezende Neta; \\ ${ }^{3}$ Grazielle Roberta Freitas da Silva; ${ }^{4}$ Telma M. Evangelista de Araújo
}

\begin{abstract}
${ }^{1}$ Mestranda em Enfermagem do Programa de Pós Graduação em Enfermagem da Universidade Federal do Piauí (UFPI). Especialista em Administração em Serviços de Saúde (Administração Hospitalar e Saúde Pública). Enfermeira da Estratégia de Saúde da Família do município de União-PI e Intensivista do Hospital Getúlio Vargas. Teresina Piauí, Brasil.

${ }^{2}$ Mestranda em Enfermagem do Programa de Pós Graduação em Enfermagem da UFPI, Docente do curso de graduação em enfermagem da Faculdade Integral Diferencial (FACID) e Enfermeira Assistencial do Hospital Getúlio Vargas. Teresina Piauí, Brasil.

${ }^{3}$ Coordenadora do curso de Mestrado do Programa de Pós Graduação em Enfermagem da UFPI. Teresina Piauí, Brasil.

${ }^{4}$ Enfermeira.Doutora em Enfermagem. Docente do Programa de Pós Graduação em Enfermagem da UFPI campus Senador Petrônio Portela-Teresina, Piauí, Brasil.
\end{abstract}

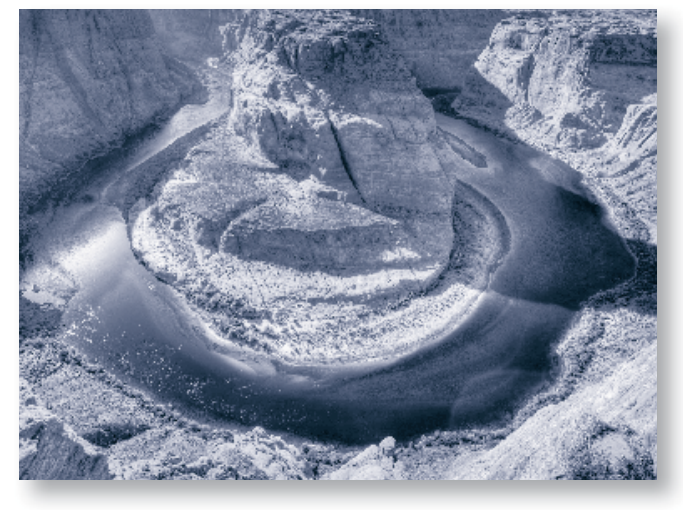

The process in Clinical Care Caritas' Jean Watson in brazilian of nursing: a systematic review

\section{ABSTRACT}

This systematic review aims at reviewing the implementation of Clinical Caritas processes, reformulation of the theory of human caring: Jean Watson, through published scientific papers. Only five products were found in the databases LILACS, SciELO, MEDLINE and BDENF after a rigorous application of inclusion judicious. The results showed that $80 \%$ of the publications are from 2006 and $60 \%$ performed at the South .The authors are post-graduate nurses who work in academy. $\mathrm{O}$ Clinical Caritas was applied in some areas of care in nursing, but there is a need for more productions to apply such an approach to care, yet little known, seeking to improve the of quality patient care and the community.

Keywords: Nursing Theory, Care, Nursing.

El proceso Clinical Caritas de Jean Watson en la enfermería brasileña: una revisión sistemática

\section{RESUMEN}

Esta revisión sistemática tiene por objetivo revisar la aplicación del proceso Clinical $\mathrm{Ca}$ ritas, la reformulación de la teoría del cuida- 
do humano: Jean Watson, a través de trabajos científico. Sólo cinco se encontraron productos en la bases de datos LILACS, SciELO, MEDLINE y BDENF después de la aplicación de rigurosos criterios de inclusión. Los resultados mostraron que el $80 \%$ de las publicaciones son de 2006 y el 60\% realizados en el Sur. Los autores son enfermeros de postgrado, que trabajan en el mundo académico. Las Clínical Caritas se aplicó en algunas áreas de la atención en la enfermería, pero no hay necesidad de una mayor producción de aplicar este enfoque a la atención, sin embargo, poco conocido, que buscan mejorar la calidad de la atención al paciente y la comunidad.

Palabras clave: Teoria de Enfermeria, Atención, Enfermería.

\section{RESUMO}

Esta revisão bibliográfica sistemática teve como objetivo analisar a aplicação do Processo Clinical Caritas, reformulação da teoria do cuidado humano de Jean Watson, através de artigos científicos publicados. Apenas cinco produções foram encontradas nas bases de dados LILACS, SciELO, MEDLINE e BDENF, após rigorosa aplicação dos critérios de inclusão. Os resultados mostraram que $80 \%$ das publicações são do ano de 2006 e 60\% realizadas na região Sul. As autoras são enfermeiras pós-graduadas que atuam na área acadêmica. O Clinical Caritas foi aplicado em algumas áreas da assistência em enfermagem, porém há uma necessidade de mais produções que apliquem essa forma de abordagem do cuidar, ainda pouco conhecida, buscando melhorar a qualidade do cuidado prestado ao paciente e a comunidade.

Palavras-chave: Teoria de Enfermagem, Cuidado, Enfermagem.

\section{INTRODUÇÃO}

Teoria é uma conceitualização articulada e comunicada da realidade inventada ou descoberta na enfermagem com a finalidade de descrever, explicar, prever ou prescrever o cuidado. Elas são essenciais na fundamentação da assistência, nas relações entre os profissionais e clientes e inter-relacionam os quatro conceitos do metaparadigma da Enfermagem: a pessoa, o ambiente/sociedade, a saúde e a enfermagem (George, 2000).

A assistência prestada pelo enfermeiro, com a evolução da enfermagem enquanto ciência, necessita de um embasamento teórico para o alcance de uma melhor qualidade de cuidado prestado ao paciente e a comunidade.

Geralmente, os profissionais da área da saúde não diferem quanto ao objeto e sujeito do cuidar/cuidado, mas sim na forma como os expressam. Mesmo que constitua um atributo para todas as áreas da saúde, no que diz respeito à Enfermagem, o cuidar/cuidado é genuíno e peculiar; e concordando com vários estudiosos no assunto, é razão existencial da Enfermagem, ficando a cura da doença como domínio da medicina (Waldow, Lopes \& Meyer,1995).

Qualquer relação entre enfermeiro-paciente pode ser considerada uma oportunidade em que o "cuidar" pode ser criado ou experimentado, dependendo da consciência, intencionalidade e filosofia (teoria) do cuidado prestado. O cuidado transcende o tempo e o espaço e continua como parte do maior complexo padrão de vida de ambos, o enfermeiro e o paciente (Watson, 2007).

É nesse contexto que as proposições das teóricas de Enfermagem auxiliam a organização e sistematização do cuidado, pois oferecem ao mesmo tempo concepções teórico-filosóficas e um método para implementar a prática. Cabe a cada enfermeiro escolher o referencial que 
melhor se articule à sua realidade para torná-lo visível e com resultados positivos ao profissional e ao cliente. Para que o enfermeiro aplique uma teoria de Enfermagem, é necessário mergulhar nas proposições, pressupostos e conceitos da teorista, interagir de forma especial e ter afinidade com a ideologia defendida (Mathias, 2006).

A escolha de uma teoria deve ser orientada por um propósito explícito ou assunto de interesse. Geralmente se escolhe trabalhar com uma teoria a partir das expectativas pessoais (Pessoa, 2006). Partindo do pressuposto de que as autoras desse estudo apresentaram aproximação com a Teoria Transpessoal de Jean Watson, bem como sua recente reformulação, o processo Clinical Caritas, e considerando que essa teoria é abordada em disciplina do mestrado do Programa de Pós-Graduação em Enfermagem da Universidade Federal do Piauí, no qual estão inseridas, surgiu o interesse de divulgar esse processo entre os enfermeiros.

A Teoria do Cuidado Humano de Jean Watson, publicada no Nursing: The philosophy and science of caring, em 1979, traz como objeto o ser humano valorizado; a saúde como uma harmonia entre a mente, o corpo e alma, e que o cuidar é transmitido através dos tempos pela cultura da profissão de enfermagem (George, 2000). A autora propõe sete pressupostos para a ciência do cuidado e dez fatores de cuidados primários para a formulação da sua estrutura. Ela considera o cuidado como um atributo mais valioso que a Enfermagem tem para oferecer à humanidade e que a doença pode ser curada por meio do cuidado (Watson, 1979).

O enfermeiro, ao escolher esta teoria como base, durante a assistência deve estar atento para sua correta aplicação bem como para o constante estudo e busca pela atualização de seus pressupostos, pois em 2005 a autora modificou a teoria, propondo um novo modelo de processo, o qual substitui os fatores da Teoria do Cuidado pelos elementos do Clinical Caritas. Ao expor estes elementos, amplia seus conceitos, incluindo a sacralidade do ser cuidado, a conexão do ser humano para um plano que extrapola o concreto e visual e a proposição do healing (recomposição, restauração, reconstituição do ser) que não deve ser entendido como cura (Mathias, 2006).

Sua teoria tem o ser humano como ponto de convergência de todas as ações de Enfermagem, a integralidade de corpo, mente e espírito em um processo transpessoal. O processo Clinical Caritas vem abordar o outro com delicadeza, com sensibilidade, dando-lhe atenção especial, exercitando uma atenção cuidadosa. Watson não explicita em sua teoria um modelo de aplicabilidade, seguindo as fases do processo de enfermagem como outras teóricas o fazem.

O processo Clinical Caritas é composto de dez elementos e os números ao lado dos elementos correspondem à ordem que a teorista propõe, no entanto não há necessidade de se seguir uma ordem, devido sua integralidade. São eles (Watson, 2005):

1. Praticar o amor, a gentileza e a equanimidade, no contexto da consciência do cuidado;

2. Ser autenticamente presente, fortalecer e sustentar o profundo sistema de crenças, mundo de vida subjetivo do ser cuidado;

3. Cultivar práticas próprias espirituais e do "eu transpessoal", ultrapassando o próprio ego;

4. Desenvolver e manter a relação de ajuda- confiança no cuidado autêntico;

5. Ser presente e apoiar a expressão de sentimentos positivos e negativos como conexão profunda com seu próprio espírito e o da pessoa cuidada; 
6. Usar-se criativamente e todas as maneiras de conhecer, como parte do processo de cuidar, engajando-se em práticas artísticas de cuidado-reconstituição;

7. Engajar-se em experiência genuína de ensino aprendizagem, que atenda à unidade do ser e dos significados, tentando manter-se no referencial do outro;

8. Criar um ambiente de reconstituição (healing), em todos os níveis, sutil de energia e consciência, no qual a totalidade, beleza, conforto, a dignidade e a paz sejam potencializados;

9. Ajudar nas necessidades básicas, com consciência intencional de cuidado, administrando o que é essencial ao cuidado humano, o que potencializará o alinhamento de corpo-mente-espírito, totalidade e unidade do ser, em todos os aspectos do cuidado;

10. Dar abertura e atenção aos mistérios espirituais e dimensões existenciais da vida-morte, cuidar da sua própria alma e da qual a do ser cuidado.

Em função de a aplicabilidade dos fatores Carative/Clinical Caritas servirem como estrutura e ordem de uma base teórico-filosófica para a disciplina e profissão de Enfermagem, faz-se necessário compreender que os ideais e os fatores de cuidado e processos propostos promoverão a evolução, o aprofundamento e a sustentação da humanidade (Watson, 2007).

Assim sendo, o objetivo desse estudo é analisar a aplicação do Processo Clinical Caritas, de Jean Watson, na assistência de Enfermagem brasileira por meio da análise das produções científicas publicadas de 2005 a 2010. Para isto, levou-se em consideração a seguinte questão norteadora: O Processo Clinical Caritas está sendo aplicado na assistência de enfermagem brasileira?

\section{METODOLOGIA}

Estudo descritivo por meio de uma revisão bibliográfica sistemática. Uma revisão sistemática, assim como outros tipos de estudo de revisão, é uma forma de pesquisa que utiliza como fonte de dados a literatura sobre determinado tema (Sampaio \& Macinni, 2007).

As amostras foram coletadas entre os meses de abril e maio de 2010, por busca de produção científica acerca do tema, selecionadas em base de dados eletrônicas, no qual foi utilizada uma amostra por conveniência. Dois revisores avaliaram independentemente os artigos selecionados. Todos os estudos identificados foram avaliados com a possibilidade de serem aprovados ou não de acordo com os critérios de exclusão e inclusão.

Os critérios de inclusão foram: artigos científicos que aplicam o processo Clinical Caritas na assistência de enfermagem brasileira; com publicação a partir de 2005 (ano que foi criado o processo Clinical Caritas) a 2010. Os descritores foram: Teoria de enfermagem; Cuidado; Enfermagem e como palavra chave Jean Watson.

A investigação se deu por meio de levantamento de produções científicas em bases de dados eletrônicas específicas da saúde, quais sejam: LILACS (Literatura Latino Americana e do Caribe em Ciências da Saúde), SciELO (Scientific Electronic Library On-line), MEDLINE (Medical Literature Analysis and Rtrieval System), BDENF (Base de dados de Enfermagem).

A avaliação dos artigos teve como base os passos da realização de avaliação crítica de literatura do JAMA (Journal of American Medical Association) que são: 1- identificação e seleção dos estudos; 2- leitura crítica (avaliação da qualidade); 3 - avaliar o resumo do artigo; 4apresentação; 5- perguntas; 6- síntese (Castro, 
2003). Contudo, foi utilizado um instrumento para análise dos estudos que consiste em um formulário para obtenção dos dados. Marconi e Lakatos (2008) definem formulário como sendo uma lista formal, catálogo ou inventário destinado à coleta de dados resultantes da observação ou do interrogatório, cujo preenchimento é feito pelo próprio investigador, à medida que faz as observações ou recebe as respostas, ou pelo pesquisado, sob sua orientação. A análise dos dados será apresentada descritivamente de acordo com os dados expostos na tabela 1 .

\section{RESULTADOS}

Após a pesquisa nas quatro bases de dados eletrônicas, e com a aplicação dos descritores e palavras-chave, analisados dentro dos critérios de inclusão e exclusão, foram encontrados cinco artigos que mostravam a aplicação do Processo Clinical Caritas na assistência de enfermagem brasileira. A LILACS foi a base eletrônica onde foi encontrado a maioria dos artigos, num total de três. Já na Medline não há presença de artigos que tragam essa temática. E os dois restantes aparecem nas bases Scielo e Bdenf (TABELA 1).

\begin{tabular}{|c|c|c|c|c|c|c|}
\hline $\begin{array}{c}\text { BASE DE } \\
\text { DADOS }\end{array}$ & ANO & LOCAL & $\begin{array}{c}\text { AREA DA } \\
\text { ENFERMAGEM }\end{array}$ & MÉTODOS & $\begin{array}{c}\text { TIPO DE } \\
\text { ESTUDO }\end{array}$ & $\begin{array}{c}\text { FORMAC̃̃o } \\
\text { DOS AUTORES }\end{array}$ \\
\hline BDENF & 2006 & RJ & Saúde da Mulher & $\begin{array}{c}\text { Análise critica de } \\
\text { CHINN-KRAMER }\end{array}$ & Descritivo & $\begin{array}{c}\text { Doutora (1) } \\
\text { Doutoranda (2) }\end{array}$ \\
\hline SCIELO & 2006 & $\mathrm{SC}$ & $\begin{array}{c}\text { Saúde do adulto e } \\
\text { do idoso. }\end{array}$ & Qualitativo & Exploratório & $\begin{array}{c}\text { Doutora (1) } \\
\text { Mestre (1) }\end{array}$ \\
\hline LILACS & 2006 & $\mathrm{SC}$ & Saúde mental & Fenomenológico & Estudo de caso & $\begin{array}{c}\text { Doutora (2) } \\
\text { Mestre (1) }\end{array}$ \\
\hline LILACS & 2006 & $\mathrm{SC}$ & $\begin{array}{c}\text { Unidade de } \\
\text { Terapia Intensiva }\end{array}$ & Qualitativo & Exploratório & $\begin{array}{c}\text { Doutora (1) } \\
\text { Mestranda (1) }\end{array}$ \\
\hline LILACS & 2007 & RJ & Hemodiálise & Qualitativo & Fenomenológico & $\begin{array}{c}\text { Doutora (1) } \\
\text { Mestre (1 }\end{array}$ \\
\hline
\end{tabular}

Tabela 1: Caracterização dos trabalhos publicados que utilizaram o processo Clinical Caritas na assistência de enfermagem brasileira. 2010.

$\mathrm{O}$ ano que mais foi publicado o assunto abordado foi 2006, com quatro artigos. Os locais de realização dos estudos se concentraram em dois estados: Rio de Janeiro e Santa Catarina, surgindo essencialmente nas regiões Sul e Sudeste brasileiras. As áreas da enfermagem se apresentaram de formas variadas, com saúde da mulher, saúde do idoso, saúde mental, terapia intensiva e hemodiálise. Em relação a abordagem metodológica dos estudos predominou a forma qualitativa com três, e o tipo de estudo na sua maioria foi exploratório (TABELA 1), sendo que apenas um artigo foi recorte de dissertação de mestrado. Destaca-se que todos os autores dos artigos analisados são do sexo feminino. Quanto a sua formação acadêmica, a maioria são doutoras em enfermagem e as demais doutorandas, mestres e mestrandas. A seguir todos esses dados apresentados de forma sucinta.

\section{DISCUSSÃO}

Observa-se que o Processo Clinical Caritas da teoria ainda é pouco explorado pelos enfermeiros e a sua aplicação na assistência de enfermagem brasileira necessita avançar, pois apenas cinco artigos que abordavam o tema foram encontrados em quatro bases de dados eletrônicas.

O número reduzido de publicações que abordam o processo Clinical Caritas e sua contribuição para a enfermagem brasileira deve-se ao fato da mudança recente da Teoria do Cuidado Humano de Jean Watson. (Fávero, Méier, Lacerda, Mazza, \& Kalinowski, 2009). Muitos estudos ainda demonstram a utilização dos fatores de cuidado ou parte deles, que são a base da Teoria Transpessoal, antes da atualização para o processo Clinical Caritas.

A utilização dos descritores é importante na busca de artigos durante uma revisão sistemática de bases eletrônica, no uso das quatro bases pesquisadas apresentou-se essencialmente a palavra cuidado. 
A necessidade de classificar as informações para futuros levantamentos em estudos e pesquisas levou a criação de descritores, também conhecidos como unitermos ou palavras-chaves (key-words), tornando mais fácil o levantamento bibliográfico. A escolha criteriosa e adequada destes unitermos é fundamental para que a indexação permita a recuperação de seus trabalhos, quando procurados pelos seus pares em uma pesquisa bibliográfica. (Oliveira, 2003).

As pesquisas encontradas foram realizadas apenas nos estados do Rio de Janeiro e Santa Catarina, provavelmente devido ao fato de que esses estados, assim como o Ceará, são os únicos locais no Brasil, que possuem conexão direta com a autora Jean Watson. Ela também visitou várias vezes a região Sul do país e atuou como parceira no programa de Pós Graduação da Região Sul, da Universidade de Federal de Santa Catarina.

A curva do número de egressos de doutorados nas duas últimas décadas é bastante satisfatória, talvez seja chegada a hora de estimular mais os estágios pós-doutorais, no País e no exterior. Há grande carência de estágios pós-doutorais no Brasil comparada às estatísticas norte-americanas. (Guimarães, 2011). Isso poderia aproximar os grupos de pesquisa em diversos paises, fazendo gerar um fluxo mais contínuo de conhecimento e experiências exitôsas de vários pesquisadores internacionais. Além disso, aproximaria os grandes teóricos de enfermagem aos estudiosos da área no Brasil.

A abordagem metodológica mais utilizada nos artigos foi a qualitativa, muito utilizada nas pesquisas de enfermagem. Esse método científico tem a função de tornar possível a abordagem da realidade a partir das perguntas feitas pelo investigador.

Em se tratando do método qualitativo, este é o que se aplica ao estudo da história, das re- lações, das representações, das crenças, das percepções e das opiniões, resultados das interpretações que as pessoas fazem a respeito de como vivem, sentem, pensam, constroem seu artefatos e a si mesmas (Minayo, 2006). Esse tipo de método de pesquisa parece ser o preferencial quando se aplica as teorias e modelos assistenciais de enfermagem.

O processo Clinical Caritas pode servir de embasamento para diferentes áreas da assistência de enfermagem, pois uma teoria de enfermagem pode ser implementada nas instituições e serviços de saúde, com implantação do processo de enfermagem, em todas as suas fases, sendo adequado de acordo com a teoria escolhida. A própria Jean Watson afirma que a ciência do cuidado propõe que a enfermagem, individual e coletivamente, contribua para a preservação da humanidade.

Todos os artigos são produções de interesse acadêmico e percebe-se que na formação das autoras há um predomínio de doutoras em enfermagem. Santos (2007) cita que o os cursos de pós-graduação contribuíram para a constituição do hábito científico das enfermeiras, mediante a concentração dos esforços individuais no sentido de realizar uma atividade de pesquisa como requisito necessário à obtenção da titulação requerida, caracterizando assim, o estreito vínculo entre a pós-graduação e o desenvolvimento da pesquisa em enfermagem no Brasil. Isso mostra que a aplicação dessa teoria ainda concentra-se em trabalhos vinculados a área da pesquisa acadêmica.

Porém é interessante ressaltar que, a realização da pesquisa científica, através da observação do pesquisador sobre a realidade, tem como objetivo responder perguntas para a construção de novos conhecimentos. No que concerne ao pesquisador, esse pode aprimorar seus conhecimentos no que diz respeito à 
ciência e ao método científico propriamente dito. Referente a sociedade, as respostas aos questionamentos dos pesquisadores podem gerar melhoria de condições de vida. Para a comunidade científica, essa terá em suas mãos resposta a um questionamento anteriormente não respondido e através dele poderá realizar novos questionamentos, além disso, enquanto enfermeiro poderá aplicar e testar o novo conhecimento gerado na sua prática.

\section{CONCLUSÃO}

Este estudo apontou que há uma necessidade de mais produções brasileiras que apliquem o processo Clinical Caritas, bem como a própria teoria de J. Watson, como forma de abordagem do cuidar, ainda pouco utilizada na prática assistencial, buscando estimular sua implantação nos serviços de saúde e melhorar a qualidade da assistência de enfermagem prestada ao paciente e a comunidade no Brasil.

\section{REFERÊNCIAS}

- Castro, A.A. (2003): Avaliação crítica de literatura:roteiro para sessão de artigos e revistas. Disponível em: <http:// www.hccpg.rn.gov.br/downloads/artigos/MEDICINA_ BASEADA_EM_EVIDENCIAS/avaliacaocriticadaliteratura.pdf $>$. Consultado em 18/01/2011.

- Castro, A.A. (2001): O que é necessário para fazer uma revisão sistemática.Disponível em: $<$ http://metodologia. org/wp-content/uploads/2010/08//lv5_rs103.PDF > . Consultado em18/01/2011.

- Fávero, L.; Méier, M.M.; Lacerda, M. R.; Mazza, V. A.; Kalinowski, L.C. (2009): Aplicação da Teoria do Cuidado Transpessoal de Jean Watson: uma década de produção brasileira. Acta paul. enferm. 22(2), 213-8.

- George, J.B. (2000): Teorias de Enfermagem: os fundamentos à prática profissional. Artes Médicas. Porto Alegre.

- Guimarães, R. (2011): Desafios da pós-graduação em saúde humana no Brasil. Rev Saúde Publica 45(1), 1-13
- Minayo, M.C.S. (2006): O desafio do conhecimento: pesquisa qualitativa em saúde. São Paulo: Hucitec,

- Marconi, M.A; Lakatos, E.M. (2008): Técnicas de Pesquisa : planejamento e execução de pesquisas ,amostragem e técnicas de pesquisa, elaboração, análise e interpretação de dados. Atlas. São Paulo.

- Mathias, J.J.S. (2006): Processo clinical caritas: novos rumos para o cuidado de enfermagem transpessoal. Acta Paulista Enfermagem 19(3), 332-7.

- Oliveira, E.F.B.; Azevedo, J.L.M.C.; Fagundes, D.J. (2003): Avaliação de descritores na angiologia e cirurgia vascular em artigos publicados em dois periódicos nacionais. Acta Cir. Bras.18(1), 62-72.

-Pessoa, S.M.F.; Pagliuca, L.M.F.; Damasceno, M.M.C. (2003):Teoria do cuidado humano: análise crítica e possibilidades de aplicação a mulheres com diabetes gestacional. Revista Enfermagem UERJ 14(3), 87-92.

- Sampaio, R.F.; Mancini, M.C. (2007): Estudos de Revisão Sistemática: um guia para síntese criteriosa da evidência científica. Revista Brasileira de Fisioterapia 11(1),83-89.

- Santos, T.C.F.; Gomes, M. L.B. (2007): Nexos entre pós-graduação e pesquisa em Enfermagem no Brasil. Rev. bras. enferm. 60(1), 91-95.

- Waldow, V.R.; Lopes, M.J.M.; Meyer, D.E. (1995): Maneiras de cuidar, maneiras de ensinar: a enfermagem entre a escola e a prática profissional. Artes Médicas. Porto Alegre:

- Watson, J. (1979): Nursing : the philosophy and science of caring. Litte Brown. Boston.

- Watson, J. (2005): Caring science as sacred science. FA.Davis. Philadelphia.

- Watson, J. (2007): Watson's theory of human caring and subjective living experiences: carative factors/caritas processes as a disciplinary guide to the professional nursing practice. Texto Contexto Enfermagem 16(7), 12935. 